\title{
Ventral hernia patient outcomes postoperatively housed on surgical vs non-surgical units
}

\author{
Elizabeth Bittner ${ }^{1} \cdot$ Walker Ueland ${ }^{1} \cdot$ Michael J. Nisiewicz ${ }^{1}$. Hussain Siddiqi ${ }^{1} \cdot$ Margaret A. Plymale $^{2}$. \\ Daniel L. Davenport ${ }^{3} \cdot$ John Scott Roth ${ }^{2}$ (1)
}

Received: 2 April 2020 / Accepted: 15 July 2020 / Published online: 27 July 2020

○) Springer Science+Business Media, LLC, part of Springer Nature 2020

\begin{abstract}
Background Inpatient hospital units vary in staffing ratios, monitoring, procedural abilities, and experience with unique patients and diagnoses. The purpose of this study is to assess the impact of patient cohorting upon ventral hernia repair outcomes.

Methods An IRB-approved retrospective review of open ventral hernia repairs between August 2013 and July 2017 was performed. The information of all patient locations during hospitalization, time at location, post-anesthesia care unit duration (PACU), and intensive care unit (ICU) duration was collected. Patient demographics, comorbidities, operative details, cost, and patient outcomes were analyzed. Multivariable analysis of log length of stay (LOS) was assessed with adjustment for clinical and operative factors.

Results 235 patients underwent open ventral hernia repair. 179 patients were admitted to surgical units, 33 non-surgical units, and 23 stayed on both units. Clinical characteristics including patient age, gender, BMI, and medical comorbidities were similar between patients boarded on surgical versus non-surgical units. Hernia, wound, and operative data were also statistically similar. Patients admitted to non-surgical units for any duration experienced longer hospital stay (4 vs. 6 days, $p<0.001$ ). Patients housed on a non-surgical unit were more likely to transfer rooms than patients on surgical units, $42.9 \%$ vs. $10.1 \%(p<0.001)$, respectively. Multivariable analysis of natural log-transformed LOS showed any stay on a non-surgical unit increased LOS by 1.0 days ( $95 \% \mathrm{Cl} 0.9-1.2$ days, $p=0.026$ ). There were no differences in ICU or PACU stay, cost, or postoperative complications in patients housed on surgical versus non-surgical units.

Conclusions Postoperative surgical patients had an increased length of stay when admitted to non-surgical units. More frequent room transfers occurred in patients admitted to non-surgical units. Evaluation of patient outcomes and LOS in open ventral hernia repair patients based on hospital unit is unique to this study.
\end{abstract}

Keywords Ventral hernia repair $\cdot$ Surgical unit $\cdot$ Cohorting $\cdot$ Length of say

To be presented as an ePoster at the 2020 Annual Meeting of the Society of Gastrointestinal and Endoscopic Surgeons, Cleveland, $\mathrm{OH}$.

John Scott Roth

s.roth@uky.edu

1 University of Kentucky College of Medicine, Lexington, KY, USA

2 Division of General Surgery, University of Kentucky Department of Surgery, UK HealthCare, 800 Rose Street, Lexington, KY 40536, USA

3 University of Kentucky Department of Surgery, Lexington, KY, USA
Ventral hernia repair (VHR) is a common surgical procedure with over 350,000 performed per year within the United States with costs exceeding $\$ 3$ billion annually [1]. Despite the frequency of these procedures and efforts to perform optimization, hernia repairs are often performed at a financial loss [2]. With aging populations and rising obesity rates, repairs are anticipated to increase in frequency. In the pursuit of financial viability and optimized patient care, several studies have identified factors that are predictive of hernia costs [2-7].

Patient characteristics and comorbidities are commonly cited influences in patient outcomes. Age, obesity, and smoking status are widely recognized to significantly impact recovery and complication rates [8-10]. Patient efforts to 
optimize comorbidities prior to hernia repair are generally advocated to improve outcomes. Surgeons may impact outcomes through the utilization of enhanced recovery after surgery (ERAS) protocols to impact both the economics and quality of care. Enhanced recovery protocols for surgery aim to address preoperative comorbidities and intraoperative interventions, as well as postoperative care to reduce variability in care resulting in reduced complications, length of stay, and costs [11-14].

While inpatient status has been shown to be a predictor of cost and appears to make little difference in complication rates and hernia recurrence compared to outpatient status [15], the influence of the quality of postoperative care has yet to be appreciated. ERAS protocols attempt to standardize postoperative care, but audits of compliance demonstrate variability in care delivery [14]. Inpatient units vary greatly in their degree of training, procedural ability, and experience with postoperative patients, and the effects of these differences in the setting of VHR have not been studied. The purpose of this study was to evaluate the impact of postoperative hospital units, including medical, surgical, and intensive care, upon hospital length of stay and early clinical outcomes of VHR patients.

\section{Materials and methods}

After receiving IRB approval, the open VHR database from the University of Kentucky between August 2013 and July 2017 was reviewed. Patient records were reviewed for demographics, comorbidities, operative details, ERAS compliance, and early patient outcomes [14]. Patient outcomes included cellulitis, infected seroma, superficial surgical site infection (SSI), deep SSI, and readmission within 30 days. Early patient stay information was collected, including patient location, room or facility transfer, hospital length of stay (LOS), PACU LOS, ICU stay, and/or ICU LOS. Patient location included facility and stay on surgical or non-surgical unit. Surgical units were defined as units primarily boarding surgical patients and staffed by healthcare workers experienced with postoperative patient care. Patient hospitalizations on non-surgical units were defined as those boarding in a hospital unit other than surgical for any duration of the hospital stay. Unit selection was based primarily on bed availability, with the exception of patients admitted to ICUs for comorbidities or complications. Room transfer was defined as any room transfer of patient, including transfers within surgical or non-surgical units, between units, or between levels of care (floor and ICU). Facility transfer was defined as transfer between the two facilities within the healthcare system. Location transfers were primarily based on bed availability, with the exception of transfer between floor units and ICUs. ICU stay includes patients admitted to both surgical and non-surgical ICU units. The surgical team was the primary team for patients on surgical floor, surgical intensive care, or non-surgical floor units. Patients admitted to non-surgical ICUs were managed by the intensivist and surgical team per hospital policy. Statistical analysis was conducted with the following two groups: patients boarded solely on a surgical unit versus boarded solely/partially on a non-surgical unit. Patients who stayed only on a non-surgical unit were combined with patients who stayed on both units for analysis. Bivariate analysis was conducted between the groups for clinical and cohorting details, operative factors, cost, and postoperative outcomes. Multivariable analysis of the natural $\log$ of the LOS in days was performed with and without adjustment for clinical, lodging, hernia, and operative factors that differed by cohorting status $(p<0.20)$. Adjusting factors were room transfer, ICU stay, ERAS protocol period, BMI, diabetes, COPD, prior hernia repairs, concomitant procedure, mesh type, and operative duration.

\section{Results}

235 patients underwent ventral hernia repair between August 2013 and July 2017. Of these, 179 (76.2\%) patients were postoperatively boarded exclusively on surgical units, while $56(23.8 \%)$ were admitted to non-surgical units. Among the 56 patients, 23 patients $(41.1 \%)$ were transferred between units (Table 1). There were no significant differences found in clinical characteristics of patients housed on surgical vs non-surgical floors including age, gender, smoking status, BMI, and medical comorbidities (Table 2). Hernia characteristics including number of prior hernia repairs, history of infected mesh, and history of abdominal wall infection did not differ between groups. Furthermore, there were no differences in operative characteristics between groups, including incidence of component separation, concomitant procedures, duration of operation, estimated blood loss (EBL), wound class, and mesh size/type (Table 3).

Length of stay in the postoperative anesthesia care unit, ICU stay, and incidence of inter-facility transfer did not

Table 1 Patient lodging and ERAS status by cohorting status

\begin{tabular}{llrlrrr}
\hline Variable & \multicolumn{2}{l}{ Surgical unit } & \multicolumn{2}{l}{$\begin{array}{l}\text { Non-surgical } \\
\text { unit }\end{array}$} & Difference \\
\cline { 2 - 3 } & $n$ & $\%$ & & $\%$ & $p$-value \\
\hline No. of patients & 179 & & & 56 & & \\
Any ICU stay & 15 & 8.4 & & 10 & 17.9 & 0.078 \\
Transferred facilities & 4 & 2.2 & & 0 & 0.0 & 0.575 \\
Room transfer & 18 & 10.1 & & 24 & 42.9 & $<0.001$ \\
Overnight in PACU & 28 & 15.6 & 7 & 12.5 & 0.670 \\
Post-ERAS protocol & 92 & 51.4 & 18 & 32.1 & 0.014 \\
\hline
\end{tabular}


Table 2 Patient demographics and clinical characteristics

\begin{tabular}{|c|c|c|c|c|c|}
\hline \multirow[t]{2}{*}{ Variable } & \multicolumn{2}{|c|}{ Surgical unit } & \multicolumn{2}{|c|}{$\begin{array}{l}\text { Non-surgical } \\
\text { unit }\end{array}$} & \multirow{2}{*}{$\begin{array}{l}\text { Difference } \\
p \text {-value }\end{array}$} \\
\hline & $n$ & $\%$ & $n$ & $\%$ & \\
\hline Age, mean $\pm S D$ & $54 \pm 13$ & & $56 \pm 11$ & & 0.290 \\
\hline $\mathrm{ASA}^{\mathrm{a}}$ class III or IV & 118 & 65.9 & 39 & 69.6 & 0.630 \\
\hline Female gender & 90 & 50.3 & 27 & 48.2 & 0.878 \\
\hline Smoking status & & & & & 0.708 \\
\hline Never & 81 & 45.3 & 22 & 39.3 & \\
\hline Former & 82 & 45.8 & 28 & 50.0 & \\
\hline Current & 16 & 8.9 & 6 & 10.7 & \\
\hline BMI $\left(\mathrm{kg} / \mathrm{m}^{2}\right)$ & & & & & 0.153 \\
\hline $23-29.9$ & 60 & 33.5 & 17 & 30.4 & \\
\hline $30-39.9$ & 106 & 59.2 & 30 & 53.6 & \\
\hline $40+$ & 13 & 7.3 & 9 & 16.1 & \\
\hline Diabetes & 41 & 22.9 & 20 & 35.7 & 0.080 \\
\hline COPD & 11 & 6.1 & 9 & 16.1 & 0.028 \\
\hline Cancer & 42 & 23.5 & 11 & 19.6 & 0.714 \\
\hline $\mathrm{CAD}$ & 22 & 12.3 & 10 & 17.9 & 0.371 \\
\hline HTN & 110 & 61.5 & 38 & 67.9 & 0.430 \\
\hline $\begin{array}{l}\text { Preoperative opioid } \\
\text { use contraindicating } \\
\text { entereg }\end{array}$ & 83 & 46.4 & 31 & 55.4 & 0.284 \\
\hline
\end{tabular}

$C O P D$ chronic obstructive pulmonary disease, $C A D$ coronary artery disease,; HTN, hypertension

aAmerican Society of Anesthesiologists' class

vary between groups. Patients housed on non-surgical units experienced more frequent room transfers, either between or within units, than patients solely in surgical units (42.9\% vs $10.1 \% p<0.001)$.

The median LOS for a patient was longer in patients admitted to non-surgical units relative to surgical units (6 days (CI $4.3-8$ days) vs 4 days (CI 3.5-6 days), $p<0.001$ ). Multivariable analysis of log-transformed LOS with adjustment for all patient, lodging, and operative factors showed an increased LOS by 1.0 days (95\% CI 0.9-1.2 days, $p=0.026$ ) among patients admitted to non-surgical units. Analysis of total cost, with or without adjustment, did not vary based on boarding location. Boarding on a non-surgical unit was not associated with a difference in early postoperative complications, including cellulitis or wound complications, or rate of readmission (Table 4).

\section{Discussion}

Inpatient units vary significantly in their staff relationships, familiarity with procedures and protocols, and training experience with categories of patients. Cohorting patients on qualified units has the potential to improve patient care, outcomes, and staff operations. However, few investigations have been conducted on patient cohorting, especially with general surgery patients. The existing cohorting research in other specialties is promising and encourages serious consideration of cohorting for general surgery patients.

One trauma center found cohorting trauma patients to one unit with daily interdisciplinary rounds resulted in significantly $(p<0.05)$ improved perceptions of teamwork, communication, and compliance with standards of care [17]. Familiarity with tasks associated with trauma patient care also increased, though it was not found to be statistically significant. Moreover, it has been shown that cohorted surgical ICU patients are seen earlier and longer on rounds than those boarded on non-surgical ICUs [18], which may result in a higher quality of care.

In addition to patient care and staff familiarity, cohorting patients also has a significant impact on outcomes and complications. When comparing surgery critical care patients, those housed on non-surgical ICUs were found to have increased rates of complications when compared to patients housed on surgical ICUs [16]. Increased complications included aspiration pneumonia $(2.2 \%$ vs $3.6 \%, p<0.01)$ and delirium ( $3.3 \%$ vs $8.3 \%, p<0.01)$. Moreover, complications not only increased on non-surgical ICUs but were more frequent the farther the non-surgical ICUs were from the surgical ICU. Aspiration pneumonia (odds ratio [OR], $2.39 ; p=0.01$ ), ventilator-associated pneumonia ( $\mathrm{OR}, 4.49$, $p<0.05)$, and delirium (OR, 6.09, $p<0.01)$ all occurred at higher rates when patients were housed farther from the surgical ICU.

Thus, while data have shown the benefits of cohorting in critically ill surgery patients, the effects on general surgery patients outside of the ICU have yet to be explored. Our analysis encourages postoperative cohorting exclusively on surgical units to significantly reduce LOS and room transfer rates $(p<0.001)$. Our study did not find an increased risk of early postoperative complications, including cellulitis and wound complications, with patients housed on non-surgical units. While no association between cost and patient location was found in our study despite LOS variation, prior findings have correlated prolonged LOS to net loss of revenue $[2,14]$. Moreover, transfer between units may increase non-surgical staff stress due to lack of experience with postoperative patients on medical units, such as seen in nursing staff caring for trauma patients prior to cohorting [17].

While our study emphasizes the need for postoperative cohorting on surgical units, lack of bed availability and overflow may result in boarding in the PACU or a non-surgical unit [16]. In an effort to improve hospital throughput, patients may be located in any area of the hospital in order to vacate beds in the PACU to avoid disrupting the flow of the operating room. The rationale for the increased rate of transfers between units is not fully understood based 
Table 3 Perioperative characteristics

\begin{tabular}{|c|c|c|c|c|c|}
\hline \multirow[t]{2}{*}{ Variable } & \multicolumn{2}{|c|}{ Surgical unit } & \multicolumn{2}{|c|}{ Non-surgical unit } & \multirow{2}{*}{$\begin{array}{l}\text { Difference } \\
p \text {-value }\end{array}$} \\
\hline & $n$ & $\%$ & $n$ & $\%$ & \\
\hline Prior hernia repair(s) & & & & & 0.067 \\
\hline 0 & 89 & 49.7 & 18 & 32.1 & \\
\hline 1 & 58 & 32.4 & 24 & 42.9 & \\
\hline $2+$ & 32 & 17.9 & 14 & 25.0 & \\
\hline Prior infected mesh & 22 & 12.3 & 9 & 16.1 & 0.499 \\
\hline Preop open wound & 14 & 7.8 & 6 & 10.7 & 0.583 \\
\hline Prior abdominal wall infection & 55 & 30.7 & 23 & 41.1 & 0.193 \\
\hline Component separation & 153 & 85.5 & 51 & 91.1 & 0.368 \\
\hline Concomitant procedure & 51 & 28.5 & 24 & 42.9 & 0.050 \\
\hline Duration of operation, mean mins. \pm SD & $196 \pm 66$ & & $213 \pm 57$ & & 0.086 \\
\hline EBL, mean CC's \pm SD, $n=158,48$ & $169 \pm 105$ & & $184 \pm 95$ & & 0.376 \\
\hline Wound class & & & & & 0.634 \\
\hline Clean & 147 & 82.1 & 42 & 76.4 & \\
\hline Clean/contaminated & 10 & 5.6 & 3 & 5.5 & \\
\hline Contaminated & 13 & 7.3 & 5 & 9.1 & \\
\hline Dirty/infected & 9 & 5.0 & 5 & 9.1 & \\
\hline Mesh size, mean $\mathrm{cm}^{2} \pm \mathrm{SD}$ & $755 \pm 429$ & & $769 \pm 361$ & & 0.823 \\
\hline Highest mesh type & & & & & 0.178 \\
\hline 1 Synthetic & 92 & 51.4 & 26 & 46.4 & \\
\hline 2 Biologic & 11 & 6.1 & 8 & 14.3 & \\
\hline 3 Bioresorbable & 76 & 42.5 & 22 & 39.3 & \\
\hline
\end{tabular}

EBL estimated blood loss
Table 4 Postoperative clinical outcomes, length of stay, and readmission rates

\begin{tabular}{|c|c|c|c|c|c|}
\hline \multirow[t]{2}{*}{ Variable } & \multicolumn{2}{|c|}{ Surgical unit } & \multicolumn{2}{|c|}{$\begin{array}{l}\text { Non-surgical } \\
\text { unit }\end{array}$} & \multirow{2}{*}{$\begin{array}{l}\text { Difference } \\
p \text {-value }\end{array}$} \\
\hline & $n$ & $\%$ & $n$ & $\%$ & \\
\hline $\begin{array}{l}\text { Median LOS days, } \\
\text { IQR }\end{array}$ & $4(3.5-6)$ & & $6(4.3-8)$ & & $<0.001$ \\
\hline Cellulitis & 8 & 4.5 & 1 & 1.8 & 0.691 \\
\hline $\begin{array}{l}\text { Wound complication, } \\
\text { any of the below }\end{array}$ & 51 & 28.7 & 17 & 30.4 & 0.866 \\
\hline Infected seroma & 18 & 10.1 & 6 & 10.7 & 1.000 \\
\hline Superficial SSI & 29 & 16.3 & 11 & 19.6 & 0.547 \\
\hline Deep SSI & 5 & 2.8 & 1 & 1.8 & 1.000 \\
\hline $\begin{array}{l}\text { Readmission w/in } \\
30 \text { days }\end{array}$ & 31 & 17.4 & 9 & 16.1 & 1.000 \\
\hline
\end{tabular}

LOS length of stay, SSI surgical site infection

upon this study. Possible explanations include transfer to accommodate other disease-specific patients on the unit or an attempt to transfer the patient to the surgical unit. Accordingly, patients may be transferred due to either hospital bed needs or the request of the surgical team. This study was not able to clearly delineate the rationale for each transfer and as a result we were not able to discern the impact of the rationale for transfer upon outcomes. Nevetheless, patients undergoing room transfer experienced increased LOS without altering clinical outcomes. In situations when surgical cohorting is not possible, interdisciplinary awareness and protocols may be needed to guarantee equivalent patient care. ERAS protocols allow providers to optimize patient care while providing nonsurgical staff with a framework to adequately treat postoperative patients on their unit. Moreover, implementation of ERAS protocol in our own institution was found to contribute to an overall $15 \%$ ( 0.7 days) reduction in mean LOS among postoperative VHR patients [14].

Limitations of this study include sample size, which may have limited statistical analysis and power of the study. Patients who stayed only on a non-surgical unit were combined with patients who stayed on both units for analysis, as these individual groups were too small for separate statistically significant analysis. Moreover, increased LOS was suggestive of increased cost but did not show statistical significance. Potential confounding factors, such as number of lab and imaging tests, were not included in this dataset. Additionally, as half of the patients in the nonsurgical stay cohort underwent a concomitant procedure, the increased complexity may have contributed to the lack of cost variability despite statistically increased LOS. 


\section{Conclusion}

Postoperative VHR patients experience an increased LOS and rate of location transfer when housed on a non-surgical unit. Early VHR outcomes, including cellulitis and wound complications, were not significantly different based on patient location. Further studies are required to further understand the specific factors associated with the increased LOS. Evaluation of the effect of VHR patient cohorting on postoperative outcomes, LOS, and cost is unique to this study.

\section{Compliance with ethical standards}

Disclosures Dr. John Scott Roth has disclosures to report: consulting and grants with Becton Dickinson, consulting with Johnson and Johnson, and consulting with Miromatrix. Walker Ueland, Hussain Siddiqi, Michael J. Nisiewicz, and Drs. Elizabeth Bittner, Margaret A. Plymale, and Daniel L. Davenport have no conflicts of interest or financial ties to disclose.

\section{References}

1. Poulose BK, Shelton J, Phillips S, Moore D, Nealon W, Penson D, Beck W, Holzman MD (2012) Epidemiology and cost of ventral hernia repair: making the case for hernia research. Hernia 16:179-183

2. Reynolds D, Davenport DL, Korosec RL, Roth JS (2013) Financial implications of ventral hernia repair: a hospital cost analysis. J Gastrointest Surg 17:159-167

3. Nisiewicz MJ, Plymale MA, Davenport DL, Saleh S, Buckley TD, Hassan ZU, Roth JS (2019) Validation and extension of the ventral hernia repair cost prediction model. J Surg Res 244:153-159

4. Ecker BL, Kuo LE, Simmons KD, Fischer JP, Morris JB, Kelz RR (2016) Laparoscopic versus open ventral hernia repair: longitudinal outcomes and cost analysis using statewide claims data. Surg Endosc 30:906-915

5. Saleh S, Plymale MA, Davenport DL, Roth JS (2018) Risk-assessment score and patient optimization as cost predictors for ventral hernia repair. J Am Coll Surg 226:540-546

6. Beldi G, Ipaktchi R, Wagner M, Gloor B, Candinas D (2006) Laparoscopic ventral hernia repair is safe and cost effective. Surg Endosc 20:92-95

7. Plymale MA, Ragulojan R, Davenport DL, Roth JS (2017) Ventral and incisional hernia: the cost of comorbidities and complications. Surg Endosc 3:341-351
8. Kroese LF, Gillion JF, Jeekel J, Lange JF, Kleinrensink GJ, Members H-C (2018) Identification of risk factors for 30-day postoperative complications in patients undergoing primary ventral hernia repair: a prospective cohort study of 2,374 patients. Surgery 163:1160-1164

9. Smolevitz J, Jacobson R, Thaqi M, Millikan S, Millikan KW (2018) Outcomes in complex ventral hernia repair with anterior component separation in class III obesity patients. Am J Surg 215:458-461

10. DeLancey JO, Blay E Jr, Hewitt DB, Engelhardt K, Bilimoria KY, Holl JL, Odell DD, Yang AD, Stulberg JJ (2018) The effect of smoking on 30-day outcomes in elective hernia repair. Am J Surg 216:471-474

11. Schneeberger S, Phillips S, Huang LC, Pierce RA, Etemad SA, Poulose BK (2019) Cost-utility analysis of biologic and biosynthetic mesh in ventral hernia repair: when are they worth it? J Am Coll Surg 228:66-71

12. Joliat GR, Labgaa I, Hübner M, Blanc C, Griesser AC, Schäfer M, Demartines N (2016) Cost-benefit analysis of the implementation of an enhanced recovery program in liver surgery. World J Surg 40:2441-2450

13. Nelson G, Kiyang LN, Crumley ET, Chuck A, Nguyen T, Faris P, Wasylak T, Basualdo-Hammond C, McKay S, Ljungqvist O, Gramlick LM (2016) Implementation of E-enhanced recovery after surgery (ERAS) across a provincial healthcare system: the ERAS Alberta colorectal surgery experience. World J Surg 40:1092-1103

14. Ueland W, Walsh-Blackmore S, Nisiewicz M, Davenport DL, Plymale MA, Plymale M, Roth JS (2019) The contribution of specific enhanced recovery after surgery (ERAS) protocol elements to reduced length of hospital stay after ventral hernia repair. Surg Endosc. https://doi.org/10.1007/s00464-019-07233-8

15. Mitchell JB, Harrow B (1994) Costs and outcomes of inpatient versus outpatient hernia repair. Health Policy 28:143-152

16. Pascual JL, Blank NW, Holena DN, Robertson MP, Diop M, Allen SR, Martin ND, Kohl BA, Sims CA, Schwab CW, Reilly PM (2014) There's no place like home: boarding surgical ICU patients in other ICUs and the effect of distances from the home unit. J Trauma Acute Care Surg 76:1096-1102

17. Meyer LK, Nanassy AD, Lavella H, Arthur LG, Grewal H (2019) Cohorting trauma patients in a medical/surgical unit at a level I pediatric trauma center to enhance interdisciplinary collaboration and documentation. J Trauma Nurs 26:17-25

18. Nunn AM, Hatchimonji JS, Holena DN, Seamon MJ, Smith BP, Kaplan LJ, Martin ND, Reilly PM, Schwab CW, Pascual JL (2017) Boarding ICU patients: are our rounding practices subpar? Am J Surg 215:669-674

Publisher's Note Springer Nature remains neutral with regard to jurisdictional claims in published maps and institutional affiliations. 